\title{
NEW METHOD OF OPERATION FOR DETACHMENT OF THE RETINA WITH TEARING OF THE MACULA LUTEA*
}

\author{
BY \\ M. MĄDROSZKIEWICZ \\ Silesian Medical Academy, Zabrze, Poland
}

DETACHMENTS of the retina with a hole in the macula lutea or posterior pole are very difficult to cure by diathermy coagulation or electrolysis. To perform this operation it has been the custom to lay bare the sclera and resect the lateral rectus muscle in order to bring the posterior pole into view by rotating the eyeball. An Arruga speculum or other suitable glass spatula was then introduced, so that the region of the posterior pole could be measured with callipers and marked with indian ink, and after these preliminaries, the diathermy operation was begun. Some surgeons even resorted to resection of the lateral wall of the orbit (Krönlein's method).

This was time-absorbing and tiring, and usually provoked a marked postoperative reaction in the tissues surrounding the eyeball. The two cases described below gave me the idea of constructing a special "calibrated" electrode, and this led me to evolve a new and simpler method of approach to the macula.

\section{Preliminary Case Reports}

Case 1, a man aged 29, had a fresh traumatic retinal detachment, with a large convoluted vesicle which refused to flatten out after rest in bed. The hole in the macula lutea was round with sharp edges, as if stamped out. Two electrolytic punctures were made in the posterior pole, in order to evacuate the subretinal fluid before beginning the actual operation. To my surprise, on the second day after what I had regarded as a preliminary procedure, the retina had completely adhered. The aperture in the macula then became covered with a delicate exudate like a fine veil, and later quite disappeared.

The detachment healed completely and permanently, the visual acuity on discharge being $6 / 18$, and 5 years after the operation $6 / 12$.

This case showed me that a tear in the macula could be cured and closed by cauterization not of the macula itself but of its surroundings.

Case 2, a woman aged 52, had a traumatic retinal detachment with a hole in the macula lutea in her only remaining eye. The detachment occurred after the patient had fallen and struck her head on the pavement. A primary electrolytic-diathermy operation gave no result, but a cure was obtained by means of a special diathermy electrode, curved to fit the circumference of the eyeball. With this electrode, two cauterizations of the retina were performed in the region of the macula lutea. The detachment was permanently cured, and the visual acuity was $6 / 24$ with $-2 \mathrm{D}$ sph., $-2 \mathrm{D}$ cyl., axis $15^{\circ}$.

This electrode, although useful, did not give complete satisfaction, since the straight section of the instrument passed gradually into the curved section. For this reason I could not calculate the exact distance of the coagulation

\footnotetext{
* Received for publication December 4, 1957.
} 
from the margin of the cornea. I had to check the distance constantly with the speculum, and even had to cauterize while using the speculum, which impeded the procedure.

After many attempts, I succeeded in constructing a new electrode which I consider excellent. So that the distance of the coagulation from the margin of the cornea could be calculated more easily, I changed the shape of the electrode to a sharp right-angle, which formed a sort of elbow. In this way the length of the inner curve from the elbow to the end of the electrode can be accurately measured.

\section{The Instrument}

This electrode is made of stainless steel (Fig. 1). The straight section consists of a thicker non-insulated handle $(n)$, and a more slender insulated shaft $(p)$, which continues as far as the elbow (pr).

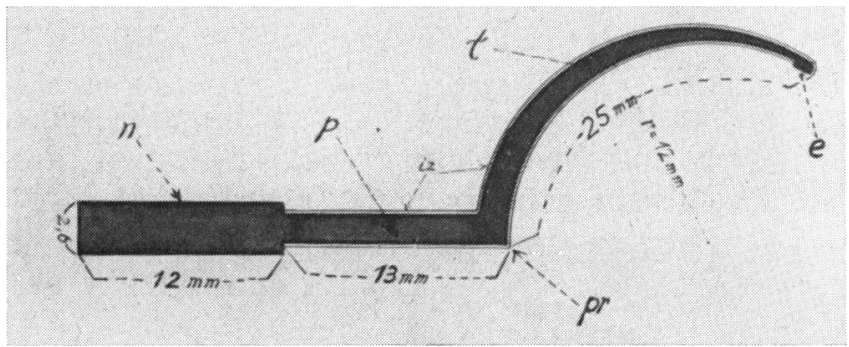

FIG. 1.-Longitudinal section of calibrated electrode, with curve $25 \mathrm{~mm}$. long.

Section $n$ is $12 \mathrm{~mm}$. long and $2.6 \mathrm{~mm}$. thick, and section $p$ is $13 \mathrm{~mm}$. long, so that the whole straight section is $25 \mathrm{~mm}$. in length. The curved insulated section of the electrode begins at right angles with the elbow ( $p r)$, and continues in a curve $(t)$, adjusted to the convexity of the sclera and gradually tapering to the end $(e)$. The inner radius $(r)$ is $12 \mathrm{~mm}$. and the length from $p r$ to $e$ is exactly $25 \mathrm{~mm}$. To avoid mistakes and for better orientation, the figure " 25 " marking the length of the inner curve of the electrode is engraved at the beginning of the handle $(n)$. The curve is so designed that the coagulation does not occur too near the macula. In transverse section, the curve of the electrode is flat-convex (Fig. 2a), the inner surface of the curve being flattened. The edges of the curved section and the end of the electrode are rounded, and the surfaces of the electrode are smoothed by japanning. At $e$, on the inner edge of the curve, is the active part of the electrode, a small non-insulated plate, projecting slightly and polished smooth, about 1 sq. $\mathrm{mm}$. in area (Fig. $2 b$ ).

Fig. 2 (a).-Transverse section of curve of calibrated electrode. plate $e$.

$2(b)$. -Tip of electrode with active
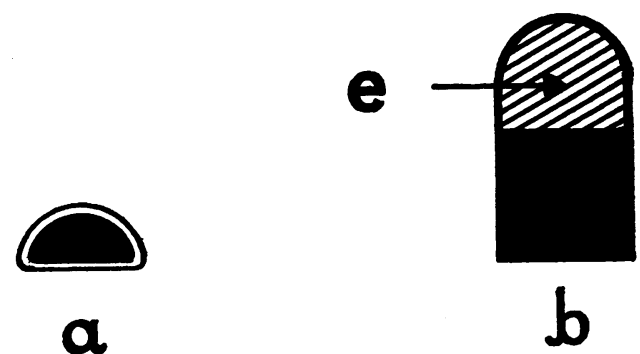


\section{Method of Use}

Approach to the Region of the Macula Lutea.-The lateral rectus muscle is not resected, but is laid bare and divided lengthwise into halves for a distance of a few millimetres. Each half is then slightly nicked at its insertion so that a small window appears in the tendon (Fig. 3). Through this, the calibrated electrode is cautiously introduced in the direction of the posterior pole, exactly in the horizontal meridian, until the elbow (pr) touches the margin of the cornea (Fig. 4).

Fig. 3.-Opening in tendon of lateral rectus muscle.
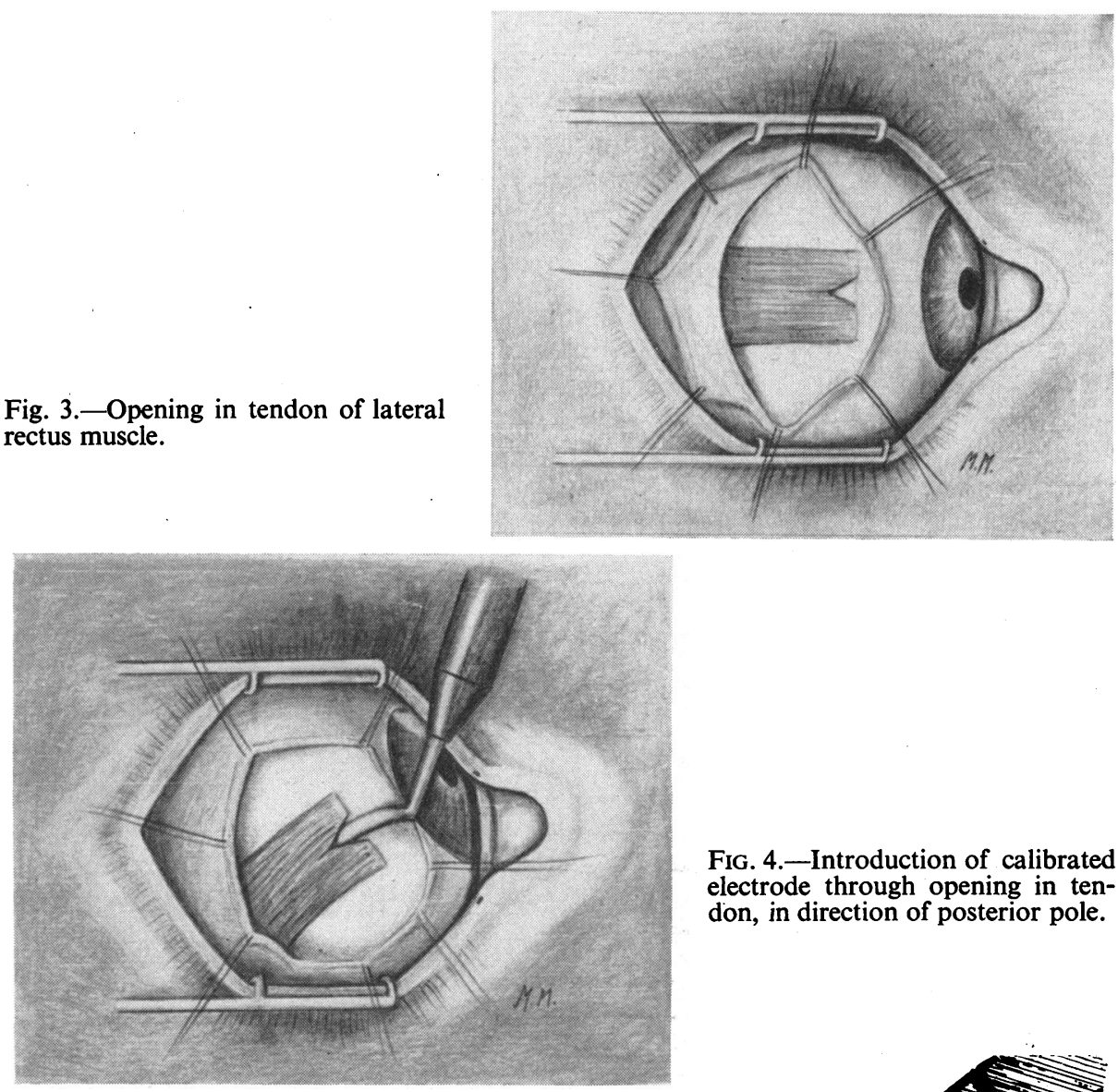

FIG. 4.-Introduction of calibrated electrode through opening in tendon, in direction of posterior pole.

The active end $(e)$ will then be in contact with the sclera in the region of the posterior pole, at a distance of exactly $25 \mathrm{~mm}$. from the margin of the cornea (Fig. 5).

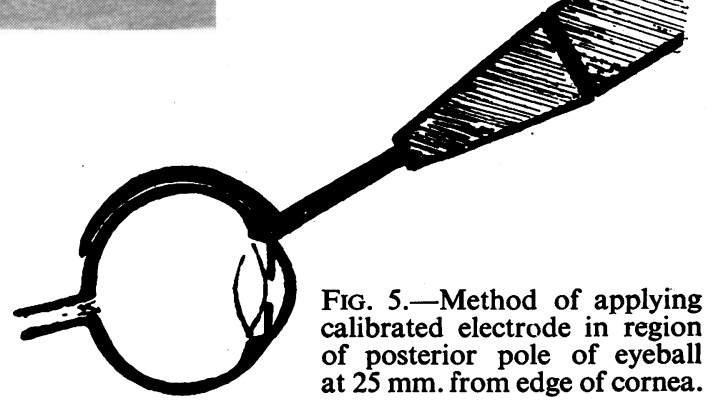


Electric Current.-As the sclera is thickest at the back of the eyeball, a brief but fairly strong current, of at least $80 \mathrm{~mA}$, is essential, since otherwise a sufficiently strong coagulation may not be achieved.

Site of Cauterization.-The electrode is aimed not at the macula lutea itself, but a little above it. When a cauterization has been performed in this position, the end $(e)$ is moved so that it makes contact a little below the horizontal meridian and a second cauterization is performed.

Even if the coagulations are not visible, owing to the size of the detachment, a closer approach to the macula is not made, especially if the eyeball is normal or the refraction is not too great. In large eyeballs with a high degree of myopia, a distance of 1 or $2 \mathrm{~mm}$. from the edge of the cornea may be marked with methylene blue; a cauterization performed with the elbow in this position will be effective at 26 or $27 \mathrm{~mm}$. from the margin of the cornea. But this means diathermy may be carried out at the back of the eyeball at a chosen distance from the limbus.

Conclusion of Operation.-If inspection with the ophthalmoscope shows the coagulations to be well placed, the subretinal fluid may be released laterally and downwards by a micropuncture or scleral trephine, and the conjunctival wound may then be sutured.

\section{Protection of the Macula from Direct Cauterization}

It has previously been the custom to cauterize the hole itself. Safar (1953), for instance, with the aid of his Ziel-Ophthalmoskop coupled with a diathermic needle electrode, cauterized the macular hole at the moment when the light of the ophthalmoscope fell upon it. Mamoli $(1936,1937)$, Bangerter (1940), and Dellaporta (1953a, b) used needles 27 to $30 \mathrm{~mm}$. long, insulated with japanning except at the extreme tip, and connected by a copper wire to the diathermy apparatus. This needle pierced the sclera in the region of the ora serrata, passed through the vitreous, and, under speculum control, reached the hole in the macula lutea for direct cautery. Bangerter (1954) has also described another method involving the surgical implantation of pieces of placenta. Meyer-Schwickerath (1956) cauterized tears in the macula by using a beam from an arc lamp, the action of which resembled that of the sun's rays on the naked eye during an eclipse (Sédan, 1956); this method caused permanent injury to the macula lutea, leaving a dark pigmented spot and central scotoma. Kettesy (1957) reported that he had cauterized a macular hole by direct solar rays; he achieved the apposition of the retina, but the visual acuity was only 0.08 after 6 weeks, and the macular vision was completely lost.

In the writer's opinion, every direct cautery of a hole in the macula lutea causes a permanent injury which is more serious than the hole itself.

It appears that macular tears do not always lie in the centre and do not cover the whole of the macula. Some of the sight cells are usually spared, and these valuable cells should not be finally destroyed by direct and violent cautery of the hole itself. 
The central area of the macula slightly exceeds the optic disc in size, the diameter of the macula being about $2 \mathrm{~mm}$. and that of the disc from 1.5 to $1.7 \mathrm{~mm}$. The diameter of the central fovea itself, on the other hand, is only 0.2 to $0.4 \mathrm{~mm}$. (Rauber and Kopsch, 1923; Eisler, 1930; KolmerLauber, 1936; Duke-Elder, 1938). The whole area of the macula, therefore, is about 3 sq. $\mathrm{mm}$., and that of the central fovea only 0.03 to $0.12 \mathrm{sq} . \mathrm{mm}$. In the structure of the macula, we distinguish the "limbus" at the circumference, where the retina is most developed, reaching a thickness of $0.49 \mathrm{~mm}$. Towards the centre we distinguish the "clivus", where the retina becomes gradually thinner; and the "fundus foveae" where the retina is only 0.075 to $0.12 \mathrm{~mm}$. thick. The fundus is slightly concave and there is also sometimes a small depression, the "foveola", with a radius of about $40 \mu$. At the central fovea the retina is composed only of closely-set cones $80 \mu$ long and 2.5 to $3 \mu$ thick. Here there may be as many as 160,000 cones to the square millimetre, whereas beyond the region of the fovea the density is about 132,000 to the square millimetre. Thus the total number of cones in the central area of the retina is about 400,000 , and that in the central fovea itself only about 16,000 . The density of the cones in this central area gives evidence of the adaptation of the macula to highly-specialized functions, and at the centre of the fovea the cones are almost completely uncovered, so that the light is unimpeded, passing through only one retinal layer.

Holes in the macula lutea are not usually large. The largest may reach one-fifth or one-sixth of the size of the disc, with an area of about $0.5 \mathrm{sq} . \mathrm{mm}$., and it may thus be assumed that the greater part of the macula is still functioning. Fortunately, such large tears are rare, and holes appear less frequently in the macula than at the periphery of the retina. The small thin central area is surrounded by the thicker limbus, which acts as a sort of protective wall to the delicate and sensitive centre. At the periphery the retina is also very thin, being only about $0.1 \mathrm{~mm}$. thick in an area extending from the ora serrata to the equator. Tears therefore occur most frequently in the anterior part of the retina, which is most exposed to tearing, whether through trauma or through degenerative changes in the form of cysts.

When, however, a macular hole does appear, a permanent loss of central vision and even complete blindness threatens, and such tears must be treated with the greatest care for conserving the remaining living cells.

According to Abramowicz (1952), the posterior pole of the eyeball is $31 \mathrm{~mm}$. from the limbus on the temporal side. The centre of the macula lutea lies somewhat towards the temporal side of the posterior pole and a little below the horizontal meridian (Rauber and Kopsch, 1923; DukeElder, 1940; Eisler, 1933; Kolmer-Lauber, 1936). It should therefore be assumed that, in the normal eyeball, the centre of the macula lutea lies about $30 \mathrm{~mm}$. from the edge of the cornea. If the elbow of the calibrated electrode described above is placed at the edge of the cornea the tip will form a coagulate $25 \mathrm{~mm}$. from the limbus. 
Experiments on rabbits (Mądroszkiewicz, 1948) showed that the action of the diathermy current is not limited to the actual site of the electrode, but always radiates 1-2 mm. from it. The effect of the calibrated electrode will thus reach as far as $27 \mathrm{~mm}$. from the edge of the cornea, leaving a space of $2 \mathrm{~mm}$. between the edge of the macula and the range of activity of the electrode. This distance is far enough to protect the macula from the harmful effects of the diathermy current, but near enough to bring about the apposition of the margin of the tear to the substratum. If the hole in the macula is very small and "punched out", the destruction of the cones may not be extensive. If it is a small slit, the edges of the hole may gape, but there will be no actual defect in the tissues, and only a few cones may have been destroyed while the majority remain unscathed. In such a case, after the retina has re-adhered, the macular vision may reach $6 / 12$ or even better.

Keeney (1955) gives a graph (Fig. 6) showing the parts of the macula on which greater or lesser degrees of visual acuity depend. Thus the loss of

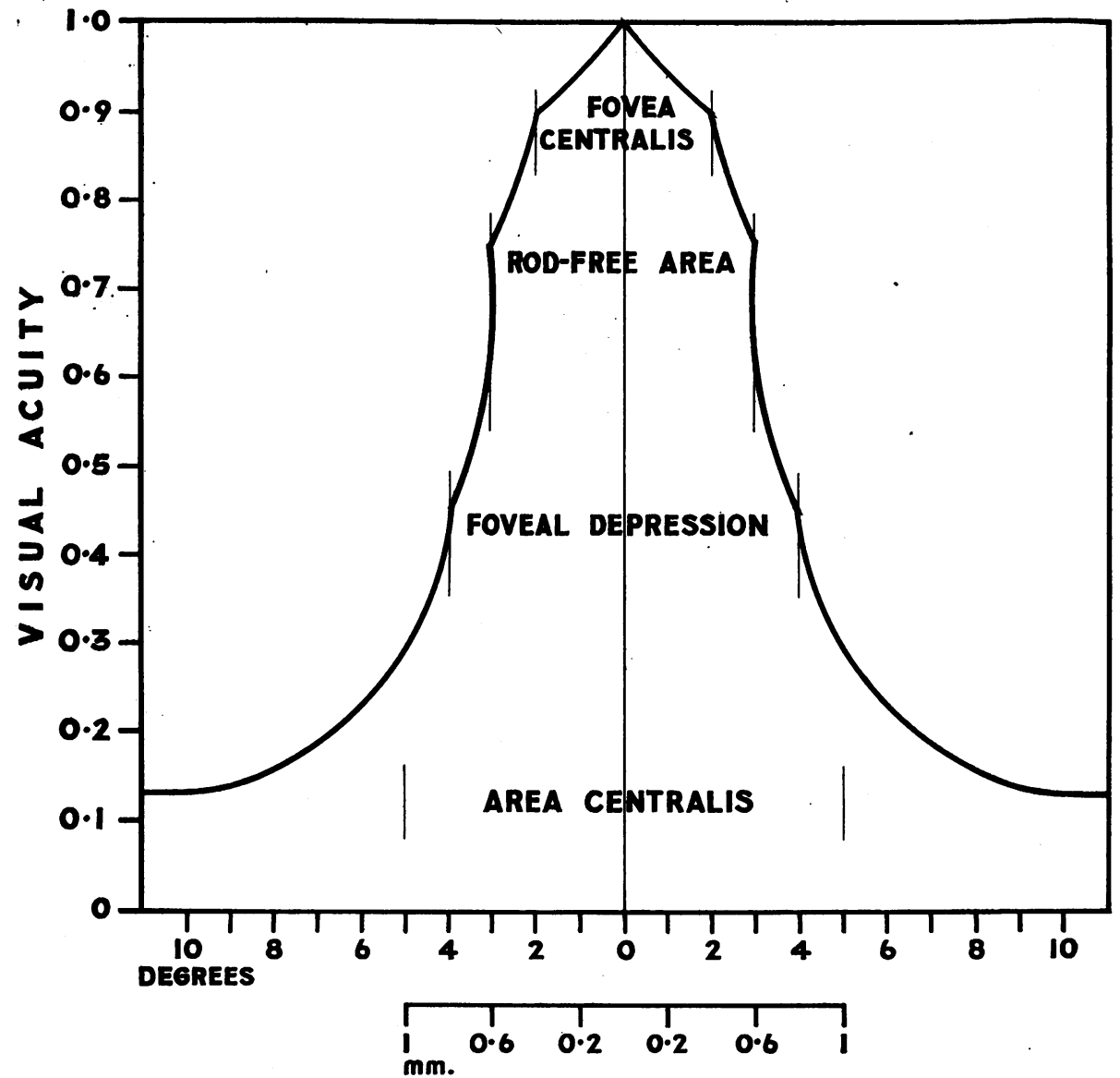

Fig. 6.-Graph (according to Keeney, 1955, p. 649, based on studies by Ludvigh, 1941), illustrating the degree of visual acuity in particular parts of the macula lutea. 
the central fovea causes the loss of the highest acuity and the visual acuity is diminished to 0.8 or 0.7 . If the greater part of the fovea undergoes destruction, the visual acuity falls to $0 \cdot 4$, and if only the limbus is left, the acuity will be only $0 \cdot 1$ or $0 \cdot 2$, after the retina has re-adhered. Below this graph I have added the distances in fractions of a millimetre, so that reckoning from the centre, each degree equals one-fifth of a millimetre. The whole macula occupies a space with a radius of $5^{\circ}$ from the centre, corresponding to a diameter of $2 \mathrm{~mm}$. A hole in the macula may be complete when the break affects the whole thickness of the retina (Fig. 7A), or it may be incomplete when either the internal limiting membrane (Fig. 7B) or the external surface remains intact (Fig. 7C).

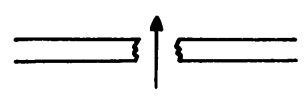

A

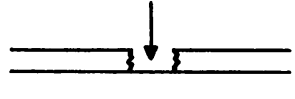

B

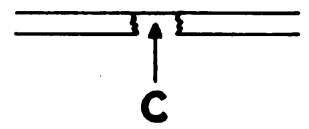

FIG. 7.-Diagram of complete (A) and incomplete (B and C) holes in the macula lutea.

At the periphery direct cautery may be performed, since the peripheral parts of the retina, especially on the temporal side or below, are not so essential for sight, but in dealing with a hole in the macula lutea it is necessary to cause the formation of inflammatory foci in the vicinity, to which the retina will adhere after the subretinal fluid has been evacuated. A hole in the macula lutea, if it has not been directly cauterized, will become completely invisible after the re-adherence of the retina. The edges will coagulate with the substratum without scar formation, and those cones which still function will be saved. Three cases are described below as examples.

\section{Case Reports}

Case 3, a woman aged 56, had received a severe blow on the head 10 days before admission to hospital; she had spent 4 days in bed, and on getting up had noticed a mist before the right eye.

On November 30,1953, the visual acuity in the right eye was $1 / 20$ with difficulty; there was no improvement with glasses, and she could not read from the chart. In the left eye it was $6 / 12$ partly, with $-1 \mathrm{D}$ cyl., axis $90^{\circ}$; for near it was 0.5 with $+2.5 \mathrm{D}$ sph., $+1 \mathrm{D}$ cyl., axis $180^{\circ}$.

The retina in the right eye was detached laterally, inferiorly, and centrally, and was convoluted. There was a small hole in the macula, a second at the meridian at 6.30 o'clock, and a third at the periphery at 7.30 o'clock, $11-12 \mathrm{~mm}$. from the limbus.

On December 11, 1953, four superficial diathermy cauterizations were performed in the vicinity of the macula (one at $26 \mathrm{~mm}$. and three at $27 \mathrm{~mm}$. from the limbus) using the calibrated electrode with a current of 60-120 mA. The peripheral tears were subjected to a few superficial cauterizations at a distance of $13 \mathrm{~mm}$. from the limbus. The subretinal fluid was evacuated by electrolytic micropunctures, and the conjunctiva was sutured. 
The post-operative course was normal. On discharge, the visual acuity was 6/18 partly; for near it was $1 \cdot 2$ with $+3 \mathrm{D}$ sph. The retina was adherent and partially pigmented, with post-operative foci near the macula and a few peripheral foci.

After 1 year and 2 months the visual acuity in the right eye was still $6 / 18$ partly; there was no improvement with glasses, and near vision was 0.6 with $+3 \mathrm{D}$ sph.

Case 4, a boy aged 19, who had been hit on the right eye with a stick, was admitted to hospital on September 29,1956 . The visual acuity in the right eye was $1 / 20$ with $+6 \mathrm{D}$. sph., and in the left $6 / 6$ partly with $+2 \mathrm{D}$ sph.

The retina in the right eye was detached centrally and superiorly, and convoluted. There was a small hole in the macula and dialysis of the retina above at 12 o'clock. On October 15, 1956, three coagulations were performed with the calibrated electrode at a distance of $25 \mathrm{~mm}$. from the limbus laterally in the vicinity of the macula, seven superficial applications surrounding the peripheral hole, and one at the vertical meridian $20 \mathrm{~mm}$. from the limbus. A micropuncture was made superiorly, through which a large quantity of subretinal fluid was evacuated.

The post-operative course was normal. On discharge on November 17, 1956, the visual acuity in the right eye was $1 / 18$ with $+6 \mathrm{D}$ sph. On February 21, 1957, it was $6 / 36$, the retina was adherent, with pigmented foci in the vicinity of the macula, and no tear was visible. A few post-operative pigmented foci were seen at the periphery.

Case 5, a man aged 54, a manual worker from Chorzów, had been operated on elsewhere in 1954 for cataract of the left eye, the sight which had been almost lost. He had worn correction - $8 \mathrm{D}$ sph. since childhood, and on April 10, 1956, he noticed that the sight of the right eye was deteriorating.

On admission to hospital on May 2,1956, the visual acuity in the right eye was counting fingers at 5 metres with $-10 \mathrm{D}$ sph., and in the left eye hand movements, not improved with glasses. The retina of the right eye was detached below both temporally and nasally, and there was a fissure-like tear in the macula. The left eye was aphakic, and the retina was almost completely detached, especially in the lower nasal quadrant. On June 6, 1956 , the lateral rectus was divided with a conical probe, a small window was made in the muscle just beside the sclera, and three superficial diathermy coagulations were performed with the calibrated electrode in the vicinity of the macula at $25 \mathrm{~mm}$. from the limbus. Three coagulations were then made on the meridians at 5.30, 7.30, and 8.30 o'clock at $20 \mathrm{~mm}$. from the limbus, a few more at $15 \mathrm{~mm}$., and nine at $9 \mathrm{~mm}$. The subretinal fluid was evacuated with a $1 \cdot 5-\mathrm{mm}$. trephine of the sclera and a puncture of the choroid with a diathermy needle.

The post-operative course was normal, the retina re-adhered, and the visual acuity on discharge was $6 / 24$ with $-8 \mathrm{D}$ sph.

In the five cases described above only single tears were found in the macula itself. In Cases 2, 3, and 4, there were also peripheral tears. In Cases 2 and 5 the retinal detachment with macular hole was unilateral.

In Cases 1, 2, 3, and 4 the macular hole was due to trauma, in Cases 1 and 4 to direct trauma to the eyeball, and in Cases 2 and 3 to indirect injury caused by a severe blow on the head. In Case 5 on the other hand, a macular hole arose without apparent cause in an eye with moderate myopia ( $-8 \mathrm{D}$ sph.) and this was the only useful eye since the other was practically blind through post-operative aphakia and a previous complete retinal detachment.

In all five cases the macular holes were complete, according to the classification of Keeney (1955). The existence of a break in the continuity of the retina was proved by the fact that there was not the slightest reaction to the 
movement of the ophthalmoscope. The presence of this reflex, according to Vogt (1936), shows that the internal limiting membrane is intact.

In all five cases the indirect method of cauterizing with a calibrated electrode gave satisfactory results and conserved the sight, and the final results were far better than in other cases described in the literature, in which the normal central (macular) vision was lost sooner or later after the operation because the hole was cauterized directly and the remaining macular cones destroyed.

Vogt (1936), who stated that both diathermy and chemical cauterization were unsuitable for macular holes because they destroyed the most valuable part of the retina, attempted to surround the hole with catholytic punctures. He achieved fairly satisfactory visual results $(0 \cdot 1$, later improved to $0 \cdot 5)$, but this was an "heroic" procedure on which not everyone might venture. He also remarked that the edges of macular holes adhered to the pigment epithelium when the coagulations were made in their vicinity; he was therefore on the right path, but his method of operation was too complicated.

From the practical point of view, the method here described shortens the time of operation (from 60 or 90 minutes to 30 minutes), and effectively achieves permanent re-apposition of the retina while sparing any central cones which may have survived the original trauma.

From this method of operation for tears in the macula, for which a calibrated electrode $25 \mathrm{~mm}$. in length has been devised, a new system of treating retinal detachment in general has been developed by the construction of additional calibrated electrodes 15 and $20 \mathrm{~mm}$. in length, which may be used according to the position of the holes. This system, which I propose to call the Polish method, will be described in detail in a future publication.

\section{Summary}

The author describes his method of treating retinal detachments with tears of the macula lutea, using a "calibrated" electrode of his own design, which produces an electro-coagulation at an accurately measured distance from the limbus without the use of a measure, callipers, or Indian ink.

The operation is performed without resecting the lateral rectus muscle, the electrode being cautiously introduced through a small opening in the muscle, and being so shaped that a diathermy coagulation can be made near the posterior pole in the vicinity of the macula, without direct injury to the macula itself.

This method is effective in achieving re-adherence of the retina without destroying any remaining macular cones.

\section{REFERENCES}

Abramowicz, I. (1952). “"Chirurgia oka w zarysie”. P.Z.W.L., Warsaw. BANGERTER, A. (1940). Ophthalmologica (Basel), 100, 351. (1954). Ibid., 127, 346. 
Dellaporta, A. (1953a). Klin. Mbl. Augenheilk., 122, 99. (1953b). Cited by Safar (1953).

DuKE-ELDER, S. (1938). "Text-book of Ophthalmology", vol. 1. Kimpton, London. (1940). Ibid., vol. 3.

- (1954). Ibid., vol. 6

Eisler, P. (1930). "Die Anatomie des menschlichen Auges", in F. Schieck and A. Brueckner, "Kurzes Handbuch für Ophthalmologie", vol. 1. Springer, Berlin.

KeENEY, A. H. (1955). Amer. J. Ophthal., 39, 648.

KeTtesY, A. (1957). Klin. Mbl. Augenheilk., 130, 465.

Kolmer-Lauber, H. (1936). "Auge", in W. von Moellendorff, "Handbuch der mikroskopischen Anatomie des Menschen"' Springer, Berlin.

LINDNER, K. (1933). Z. Augenheilk., 81, 186.

(1945). "Operative Behandlung der Netzhautabloesung", in R. Thiel, "Ophthalmologische Operationslehre", vol. 2. Thieme, Leipzig.

Ludvigh, E. (1941). Amer. J. Ophthal., 24, 303.

MaMOl, L. (1936). Atti Soc. oftal. ital., 41 (Abs. in Zbl. Ophthal., 1937, 37, 252). (1937). Ann. Oculist. (Paris), 174, 309 (Abs. in Zbl. Ophthal., 1937, 39, 409).

MádroszKIEWICZ, M. (1948). Przegl. lek., 4, 292. (1958). Klin. Oczna., 28, 71.

Meyer-Schwickerath, G. (1956). Ann. Oculist. (Paris), 189, 533.

RAuber, A., and KoPSCH, F. (1923). "Das Sehorgan', in "Lehrbuch und Atlas der Anatomie des Menschen". Thieme, Leipzig.

Safar, K. (1953). "Elektrochirurgie am Auge". Springer, Berlin.

SÉdAN, J. (1956). Ann. Oculist. (Paris), 189, 337, 602.

VoGT, A. (1936). "Die operative Therapie und die Pathogenese der Netzhautabloesung". Enke, Stuttgart. 